Pituitary iron and factors predictive of fertility status in transfusion-dependent thalassemia

Long-term survival of patients with transfusion-dependent thalassemia (TDT) raises hopes of attaining a decent quality of life, having a family and maintaining reproductive potential. However, for many these remain significant challenges despite advances in the management of iron overload. While pregnancies are possible, there is inadequate information to guide patients on their reproductive ability and on markers that predict when they will have infertility issues. Preservation of fertility and timely interventions remain pervasive concerns. Although the association of iron toxicity with cardiac and pancreatic dysfunction has been investigated extensively, the progression of iron deposition in the anterior pituitary leading to hypogonadotropic hypogonadism and infertility has not been adequately explored. ${ }^{1}$

To address these issues, we sought to investigate pituitary dysfunction and stratify the risk of infertility utilizing reproductive data and information on systemic iron load. We also evaluated the progression of pituitary dysfunction and systemic iron parameters and their effect on reproductive capacity. Magnetic resonance imaging (MRI) measurements of anterior pituitary iron (R2) and volume have established reference data in normal controls and proved an adequate method to predict clinical hypogonadism in thalassemia. ${ }^{2-4}$ Identifying risk thresholds could assist in establishing the timing and effectiveness of intensifying chelation treatment to halt further damage and potentially restore function.

The study was approved by institutional review boards and all patients provided written informed consent to participation.

Fifty-three TDT patients, 10 years and older, and 12 healthy subjects (age 12-40 years) underwent MRI (Philips Achieva $\left.{ }^{\circledast}, 1.5 \mathrm{~T}\right)$ to determine cardiac iron (T2*), anterior pituitary iron (R2) and three-dimensional volume. ${ }^{5}$ Levels of non-transferrin bound iron, labile plasma iron (LPI), and ferritin were measured in blood samples, and liver iron concentration (LIC) and myocardial iron were quantified by MRI and a superconducting quantum interference device (SQUID), respectively. In order to assess fertility, plasma gonadotropins (luteinizing hormone $[\mathrm{LH}]$ and follicular-stimulating hormone $[\mathrm{FSH}]$ ), estradiol and testosterone levels were assayed. AntiMüllerian hormone (AMH) levels were determined in women in order to evaluate ovarian reserve and inhibin $B$ levels were measured in men to assess impairment of spermatogenesis. ${ }^{6,7}$ In 17 patients these measures were repeated 2-3 years later to assess for changes over time. The patients' pubertal and reproductive history was recorded. $Z$ scores for anterior pituitary iron accumulation, $Z(R 2)$, and volume, $Z(V)$, were calculated for each patient. ${ }^{3}$ Parametric statistics were applied to characterize patients: mean \pm standard deviation, $t$-test, and Pearson correlation. Associations of Z(R2) and Z(V) with age and $\mathrm{LH}$ and FSH levels were demonstrated by boxplots and linear regression. Receiver operating characteristic (ROC) curve analysis was used in patients for categorization into $Z(R 2) \leq 4$ and $>4$ to examine the sensitivity and specificity for systemic iron parameters.

Moderate-severe pituitary iron deposition $\mathrm{Z}(\mathrm{R} 2)>2$, mean 5.4) was observed in 33 patients (62\%, age $26 \pm 7$ years), a rate comparable to that in the report by Noetzli et al. ${ }^{4}$ The rest of the patients ( $32 \%$, age $23 \pm 8$ years) had no iron accumulation (mean Z(R2) of 0.03 ). Thirty-two patients $(60 \%)$ had a $Z(V)<-2$ (mean -2.8$)$ indicating sig- nificant gland shrinkage; the remaining $40 \%$ had a $Z(V)$ of -0.7 . There was a negative correlation between $Z(R 2)$ and $\mathrm{Z}(\mathrm{V}): \mathrm{r}=-0.36, P=0.02$ (Figure $1 \mathrm{~A})$. We found that a $\mathrm{Z}(\mathrm{R} 2)$ value $>4.0$ predicted pituitary volume loss: $\mathrm{Z}(\mathrm{V})$ $<-2.0$ was present in $17 / 20$ patients with a $Z(R 2)>4$ and in only $16 / 33$ with a $Z(R 2)<4.0(P<0.009)$.

Twenty-three patients $(43 \%, 28 \pm 6$ years $)$ simultaneously had $Z(R 2)>4$ and $Z(V)<-2$, of whom ten $(43 \%)$ were splenectomized. Overall, patients who underwent splenectomy had higher pituitary R2, smaller gland volume and a significantly higher cardiac iron in comparison to the non-splenectomized patients $(P<0.004)$ while LIC was similar (Table 1). This supports the notion that

Table 1. Patients' disease characteristics.

\begin{tabular}{lc}
\hline Characteristics & $N(\%)$ or mean \pm SD \\
Study patients & $53^{*}$ \\
Females:males & $27: 26$ \\
Age (years) & $25 \pm 8$ (range $10-45)$ \\
$\beta^{0}$ thalassemia & 34 \\
E/ $\beta^{0}$ thalassemia & 18 \\
Hb H-Constant Spring & 1 \\
Years on regular transfusions & $18.7 \pm 6$ \\
$\quad \beta^{0}$ thalassemia major (mean age $\left.24 \pm 6\right)$ & $19.7 \pm 6$ \\
$\quad$ E/ $\beta^{0}$ thalassemia (mean age $\left.28 \pm 6\right)$ & $17 \pm 5$ \\
Chelation treatment & \\
$\quad$ Mono therapy (DFX) & $29(55 \%)$ \\
$\quad$ Combination therapy (DFX \& DFO) & $24(45 \%)$ \\
Systemic iron measures & $15 \pm 11$ \\
LIC (mg/g dw) & $27.2 \pm 10$ \\
Cardiac T2* (ms) & $2620 \pm 2332$ \\
Mean annual ferritin ( $\mu \mathrm{g} / \mathrm{L})$ & $1.9 \pm 1.3$ \\
NTBI $(\mu \mathrm{mol} / \mathrm{L})$ & $0.25 \pm 0.5$ \\
LPI $(\mu \mathrm{mol} / \mathrm{L})$ &
\end{tabular}

\section{Anterior pituitary}

$\mathrm{R} 2\left(\mathrm{~s}^{-1}\right)$

Z score of R2: Z(R2)

Pituitary $\mathrm{Z}(\mathrm{R} 2)>2(\mathrm{n}=33 ; 62 \%)$

Females' Z(R2)

Males' Z(R2)

Volume $\left(\mathrm{mm}^{3}\right)$

$\mathrm{Z}$ score of volume: $\mathrm{Z}(\mathrm{V})$

Pituitary $\mathrm{Z}(\mathrm{V})<-2(\mathrm{n}=32 ; 60 \%)$

Females' $Z(V)$

Males' Z(V)

\section{Spleen status}

Group I: splenectomized

Age (years)

Age at splenectomy (years)

Years since splenectomy

Z(R2) score

$\mathrm{Z}(\mathrm{V})$ score

Cardiac T2*(ms)

LIC (mg/g dw)

Mean annual ferritin $(\mu \mathrm{g} / \mathrm{L})$

Group II: non-splenectomized

Age (years)

Z(R2) score

Z(V) score

Cardiac T2* (ms)

LIC (mg/g dw)

Mean annual ferritin $(\mu \mathrm{g} / \mathrm{L})$

$14.5 \pm 3$

$3.4 \pm 3.6$

5.4

$3.2 \pm 4.4$

$3.7 \pm 4.5$

$377 \pm 110$

$-2.0 \pm 1.2$

$-2.8$

$-2.3 \pm 1.1$

$-1.7 \pm 1.3$

*Two individuals died from iron overload complications during the study period. SD: standard deviation; DFX: deferasirox; DFO: deferoxamine; LIC: liver iron concentration; dw: dry weight; NTBI: non-transferrin bound iron; LPI: labile plasma iron; $\mathrm{LIC}$ and cardiac iron (T2*) were obtained within 6 months of laboratory studies Normal NTBI and LPI: not detectable. 
splenectomy accelerates iron loading in the pituitary gland as previously reported to occur in the myocardium. ${ }^{8}$ To assess for a possible confounding effect of older age (mean $31 \pm 6$ and $21 \pm 6$ years in splenectomized versus non-splenectomized patients, respectively) a multivariable general linear model predicting $Z(V)$ was constructed. It showed that splenectomy was significant while age was not $(P=0.002$ and $P=0.218$, respectively).

Patients in the third decade of age had significantly higher pituitary iron accumulation than those in their second decade ( $Z(R 2) 4.9 \pm 3$ vs. $1.1 \pm 2, P<0.002$ ), paralleled by a decrease in pituitary volume, most apparent in the fourth decade of life (Online Supplementary Figure S1). These findings appear to be a decade later than in a prior study. ${ }^{4}$ It is possible that this difference is the consequence of improved adherence to treatment or greater efficacy of the oral chelation drug, deferasirox, used for extended periods by most of our patients. Indeed, two studies have shown stabilization of pre-existing hypogonadism, and a low rate of new endocrine disorders with long-term (5 and 6.5 years) chelation with deferasirox. ${ }^{9,10}$ However, further longitudinal study is needed to assess the effect of chelation regimens on pituitary iron.

Pituitary MRI findings in the 12 control patients were consistent with the published reference data used in this study.

The levels of gonadotropins ( $\mathrm{LH}$ and $\mathrm{FSH}$ ) were inversely correlated with pituitary iron load, Z(R2), and directly correlated with anterior pituitary volume, $\mathrm{Z}(\mathrm{V})$; LH/FSH levels declined with gland shrinkage (Online Supplementary Figure S2). Ten of 24 post-pubertal women reported regular menstrual periods (group I) and 14 had primary or secondary amenorrhea (group II). LH and FSH concentrations were normal in group I and significantly lower in group II $(P<0.04$ and $P<0.005$, respectively). Pituitary iron load and pituitary volume also differed significantly between the two groups $(P<0.03$ and $P<0.006$, respectively) (Figure 1B). These findings suggest low reproductive potential among women in group II, as also indicated by low AMH levels (Table 2), signifying compromised oocyte count ${ }^{6}$ and diminished reproductive potential. AMH, a key marker of ovarian reserve, has been reported to be positively associated with pregnancy and live birth rates after assisted reproduction. Its validity in TDT women was demonstrated and it serves as a prognostic factor for the probability of pregnancy when gonadotropin secretion is compromised. . $^{11,12}$

Men who received testosterone supplementation (group II) had lower LH and FSH levels compared to men who did not (group I); $P<0.03$ and $P<0.001$, respectively. Although testosterone treatment could contribute to the low levels, our findings indicate that the main causes are pituitary iron toxicity and volume loss since $Z(R 2)$ and $Z(V)$ differed significantly between the two groups $(P<0.02$ and $P<0.0002$, respectively). Such low FSH levels, as observed in group II (1.3 mIU), are associated with low spermatogenesis as also shown in thalassemic men with severe iron overload. ${ }^{13}$ Furthermore, the levels of inhibin B, a sensitive marker for male infertility, differed significantly between these two cohorts $(P<0.03)$ and were lower than the reported mean level in men with normal fertility parameters. The concentration of inhibin $B$ was below the $10^{\text {th }}$ percentile in $8 / 26$ men $(31 \%)$, in a range that considerably decreases the likelihood of retrieving testicular spermatozoa. ${ }^{14}$

Cardiac iron, in addition to strongly correlating with pituitary iron, had an optimal cutoff for predicting a pituitary iron threshold associated with reduced volume and loss of function; area under the curve (AUC) $=0.96$, $P=0.009$ (Figure 1C). The close association between cardiac and pituitary iron might be caused by the additional cellular iron uptake through L-type $\mathrm{Ca}^{2+}$ channels. ${ }^{15}$ Fifteen of 16 patients with a T2* $\leq 20 \mathrm{~ms}$ had a $Z(R 2)>4.0$ while only $5 / 35$ with a T2* $>20 \mathrm{~ms}$ had a $Z(R 2)>4.0$ $(P<0.006)$. Therefore, adult patients with a T2* $<20 \mathrm{~ms}$ are at risk of a decline in fertility potential, but assessing fertility markers even before this cardiac T2* threshold is recommended. LIC and ferritin concentration were less predictive of pituitary iron load; the AUC for these were 0.76 and 0.80 , respectively, possibly because of the greater variation in liver and plasma iron. We found no correlation of pituitary iron with non-transferrin bound iron or labile plasma iron.

The effect of chelation over time has not been examined before. We conducted a second measurement $(n=17)$ after an interval of $2.6 \pm 1$ years which demonstrated decreases in systemic iron: LIC by $46 \pm 20 \%$, ferritin by $44 \pm 21 \%$ and cardiac iron by $34 \pm 30 \%$ in 11,10 and 12

Table 2. Patients' fertility and hormone data.

\begin{tabular}{|c|c|}
\hline Characteristics & $\begin{array}{l}\text { N (\%) or mean } \\
( \pm S D)\end{array}$ \\
\hline Females^ & 24 \\
\hline $\begin{array}{l}\text { Group I: regular menses } \\
\text { Age (years) } \\
\text { LH/FSH (mIU/mL) } \\
\text { Estradiol (pg/mL) } \\
\text { AMH (ng/mL) } \\
\text { Pituitary Z(R2) score } \\
\text { Pituitary Z(V) score } \\
\text { Cardiac T2* (ms) }\end{array}$ & $\begin{array}{c}10(42 \%) \\
20 \pm 5 \\
8 \pm 7 ; 5 \pm 2 \\
84 \pm 75 \\
4 \pm 3.5 \\
1.3 \\
-1.7 \\
28 \pm 7\end{array}$ \\
\hline $\begin{array}{l}\text { Group II: amenorrhea } \\
\text { Age (years) } \\
\text { LH/FSH (mIU/mL) } \\
\text { Estradiol (pg/mL) } \\
\text { AMH (ng/mL) } \\
\text { Pituitary Z(R2) score } \\
\text { Pituitary Z(V) score } \\
\text { Cardiac T2* (ms) }\end{array}$ & $\begin{array}{c}14(58 \%) \\
31 \pm 5 \\
2 \pm 2 / 2.9 \pm 3 \\
40 \pm 47 \\
1.6 \pm 1 \\
5.1 \\
-2.9 \\
20 \pm 9\end{array}$ \\
\hline $\begin{array}{l}\text { Pregnancies/live children } \\
\text { With assistant reproductive therapy }\end{array}$ & $\begin{array}{c}5 / 4 \\
2\end{array}$ \\
\hline Males & 26 \\
\hline $\begin{array}{l}\text { Group I: no testosterone treatment } \\
\text { Age (years) } \\
\text { LH/FSH (mIU/mL) } \\
\text { Inhibin B (pg/mL) } \\
\text { Testosterone (ng/dL) } \\
\text { Pituitary Z(R2) score } \\
\text { Pituitary Z(V) score } \\
\text { Cardiac T2* (ms) } \\
\text { Father of a child } \\
\text { With assistant reproductive therapy }\end{array}$ & $\begin{array}{c}16(62 \%) \\
22 \pm 7 \\
3.7 \pm 2.7 / 4.5 \pm 2.4 \\
140 \pm 69 \\
385 \pm 240 \\
2.3 \\
-1.0 \\
30 \pm 7 \\
3 \\
3\end{array}$ \\
\hline $\begin{array}{l}\text { Group II: on testosterone replaceme } \\
\text { Age (years) } \\
\text { LH/FSH (mIU/mL) } \\
\text { Inhibin B (pg/mL) } \\
\text { Pituitary Z(R2) score } \\
\text { Pituitary Z(V) score } \\
\text { Cardiac T2* (ms) }\end{array}$ & $\begin{array}{c}10(38 \%) \\
28 \pm 6 \\
1.5 \pm 1.8 / 1.3 \pm 1.6 \\
80 \pm 55 \\
5.7 \\
-2.7 \\
23 \pm 8 \\
\end{array}$ \\
\hline
\end{tabular}

${ }^{\wedge}$ Levels of estradiol and testosterone from patients receiving hormone replacement were excluded from analysis. LH: luteinizing hormone; FSH: follicular-stimulating hormone; AMH: anti-Müllerian hormone. Normal ranges: $\mathrm{AMH}$ in fertile women, 1.0$4.0 \mathrm{ng} / \mathrm{ml}$; Inhibin B in adult men, $125-215 \mathrm{pg} / \mathrm{mL}$ (both change with age) 

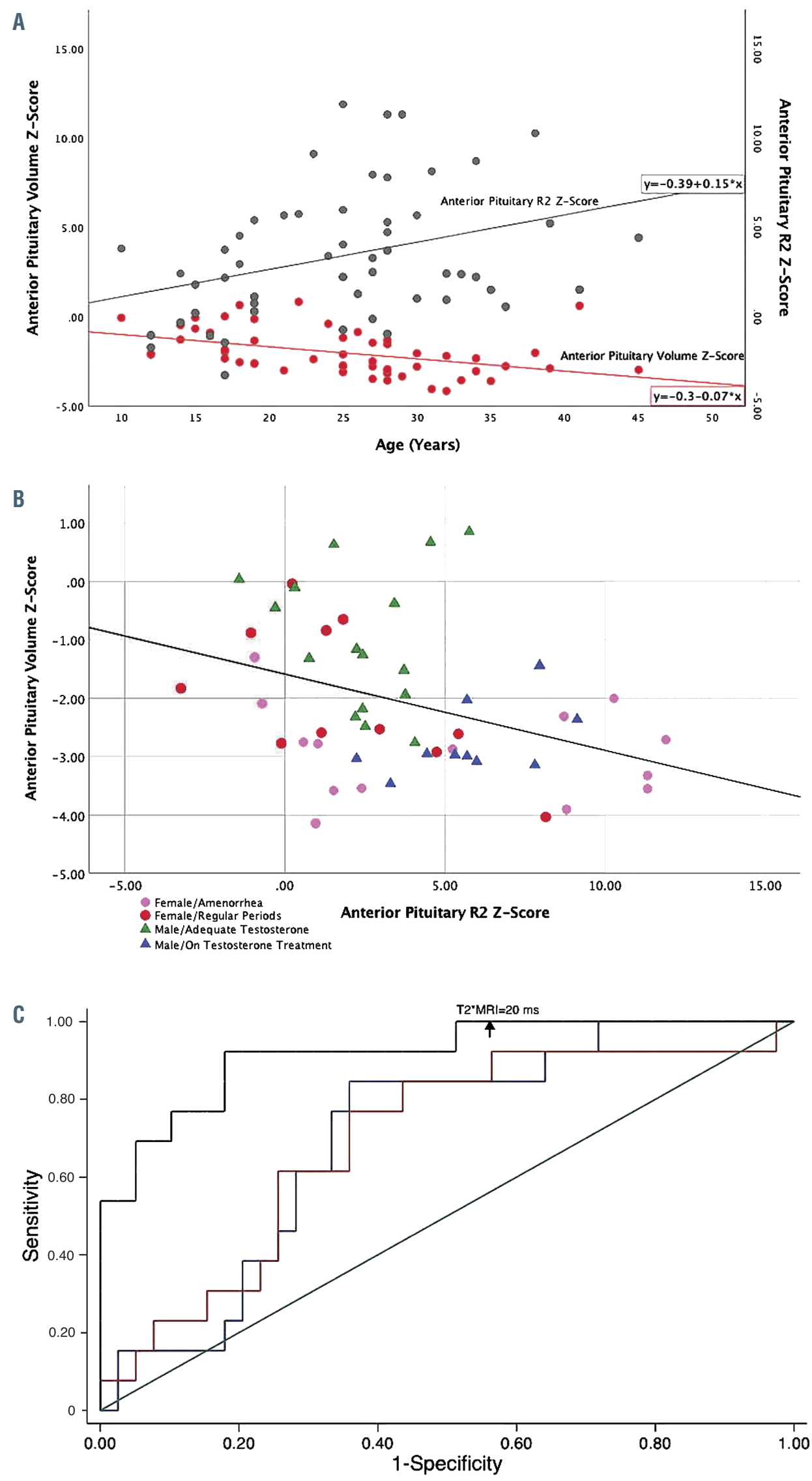

Figure 1. Pituitary iron and volume, relation to hormonal status and to systemic iron. (A) Z scores of pituitary R2 and volume $(\mathrm{V})$ as a function of age in the 53 chronically transfused patients. Pituitary iron and volume are inversely correlated $(r=-0.36 ; P<0.02)$. (B) Distribution by gender and hormonal/reproductive status (based on groups I and II shown in the Tables). The majority of group I patients (men with adequate endogenous testosterone and women with regular periods, represented as green triangles and red circles, respectively) have only moderately elevated pituitary iron accumulation and small changes in pituitary volume; $Z$ scores 0 to 5.0 and 0 to -3.0 , respectively. Men requiring testosterone treatment (blue triangles) and women with amenorrhea (pink circles) (group II) had more severe pituitary iron loading and more notable gland shrinkage. (C) Receiveroperating characteristic (ROC) curves for the prediction of pituitary iron show that cardiac T2* correlates strongly with pituitary iron. Liver iron and ferritin concentrations are weakly predictive, the cardiac $T 2 *$ is superior to both of these measures. The point of cardiac $\mathrm{T}_{2} *=20 \mathrm{~ms}$ is shown. This value was the optimal cutoff for predicting abnormal pituitary iron; ROC 
A

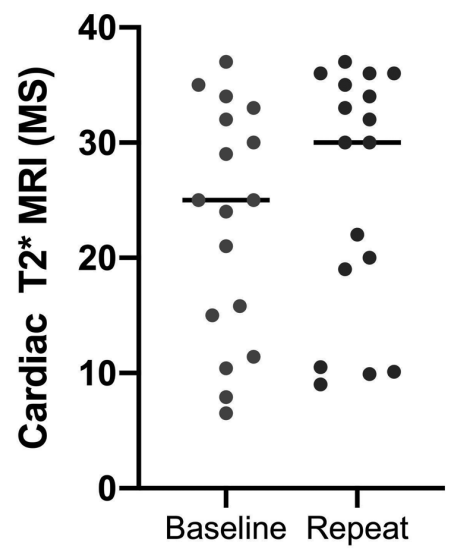

B
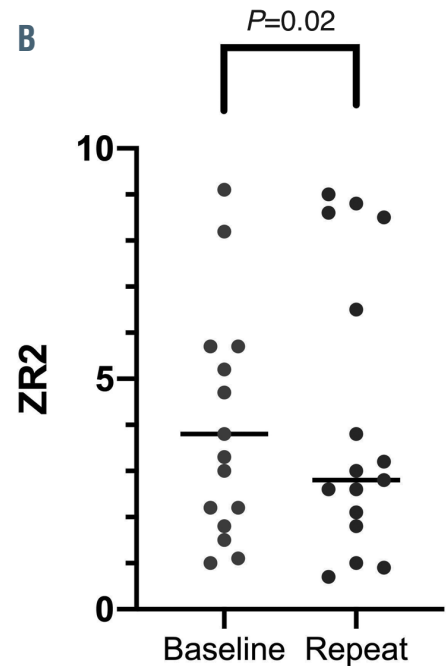

C

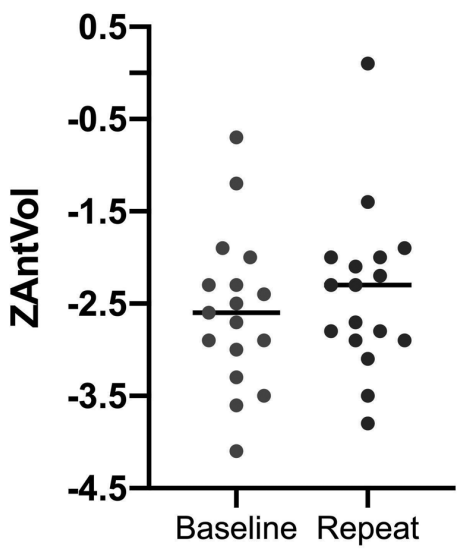

Figure 2. Effect of chelation on pituitary iron and volume. A longitudinal study (mean 2.6 years) showed a significant decrease in pituitary iron ( $P<0.02)$ correlating with an improvement in cardiac iron $(P<0.03)$. The change in pituitary volume was minimal, as expected to be irreversible, and not statistically significant.

patients, respectively. Results were stable or worse in the remaining ones. A parallel improvement in pituitary $\mathrm{Z}(\mathrm{R} 2)$ was noted in $9 / 17(53 \%)$ individuals, whose mean baseline $Z(R 2)$ was 6.4 (Figure 2). The change was modest in those with a baseline $Z(R 2)>8$, suggesting chelation failure in severe pituitary iron load. Eight of the nine patients with improved $Z(R 2)$ also had a decrease in cardiac iron, again showing a good correlation of these two measures. Pituitary volume remained abnormally low in subsequent testing. The mean $\mathrm{Z}(\mathrm{V})$ was -2.6 at baseline and -2.4 upon repeat imaging, confirming the irreversible cell destruction in patients with high iron burden. $\mathrm{LH} / \mathrm{FSH}$ concentrations decreased by a mean of $28 \%$ at repeat testing in 7/8 women. AMH levels remained stable overall, supporting reports on preserved gonadal function and successful ovulation induction despite low $\mathrm{LH}$ and FSH levels. Longitudinal studies on a larger scale are needed to further explore these findings.

In summary, our data confirm previous pituitary MRI findings of iron and volume changes and risk thresholds for hypogonadism. Our study further highlights the high prevalence and progressive nature of pituitary iron loading, despite advances in chelation therapy, causing irreversible volume loss and deleterious effects on reproductive status. Splenectomy and older age are risk factors for these effects.

Our findings also validate cardiac T2*MRI as a proxy for pituitary R2, which can be used where pituitary MRI is not available. The findings also have implications for modifying chelation treatment, which is effective in mildmoderate cases in preventing or delaying infertility. ${ }^{16}$ Discussion of reproductive status, utilizing these hormonal and MRI findings, and informing patients of higher risk of infertility are prudent for proper planning of fertility preservation.

Sylvia T. Singer, ${ }^{1}$ Roland Fischer, ${ }^{2}$ Isabel Allen, ${ }^{3}$ Ashutosh Lal, ${ }^{1}$ Elliott Vichinsky, ${ }^{1}$ Qing Yuan ${ }^{4}$ and Zhiyue J. Wang ${ }^{4}$

${ }^{1}$ Division of Hematology-Oncology, Department of Pediatrics, University of California San Francisco, UCSF Benioff Children's Hospital Oakland, Oakland, CA, USA; ${ }^{2}$ University Medical Center Hamburg-Eppendorf (UKE), Hamburg, Germany; ${ }^{3}$ University of California San Francisco, San Francisco, CA, USA and ${ }^{4}$ University of Texas Southwestern Medical Center, Texas, TX, USA
Correspondence:

SYLVIA T. SINGER - sylvia.singer@UCSF.edu

doi:10.3324/haematol.2020.252726

Received: March 23, 2020.

Accepted: October 9, 2020.

Pre-published: October 29, 2020.

Disclosures: no conflicts of interest to disclose.

Contributions: STS designed and performed the study, analyzed results and wrote the paper; ZJW and QY analyzed patients' images and pituitary data; $R F$ reviewed and analyzed the data and reviewed and revised the manuscript; IEA performed the statistical analysis; $A L$ and $E V$ reviewed and revised the paper.

\section{References}

1. Wood JC. Estimating tissue iron burden: current status and future prospects. Br J Haematol. 2015;170(1):15-28.

2. Wood JC. Use of magnetic resonance imaging to monitor iron overload. Hematol Oncol Clin North Am. 2014;28(4):747-764, vii.

3. Noetzli LJ, Panigrahy A, Hyderi A, Dongelyan A, Coates TD, Wood JC. Pituitary iron and volume imaging in healthy controls. AJNR Am J Neuroradiol. 2012;33(2):259-265.

4. Noetzli LJ, Panigrahy A, Mittelman SD, Hyderi A, Dongelyan A, Coates TD, et al. Pituitary iron and volume predict hypogonadism in transfusional iron overload. Am J Hematol. 2012;87(2):167-171.

5. Wang ZJ, Wang DJ, Chia JM, Yuan Q, Morriss MC, Rollins NK. A software tool for semi-automated quantification of pituitary volumes. International society of magnetic resonance imaging in medicine (ISMRM) 19th meeting. Montreal, Canada, 2011:4247.

6. La Marca A, Broekmans FJ, Volpe A, Fauser BC, Macklon NS. AntiMullerian hormone (AMH): what do we still need to know? Hum Reprod. 2009;24(9):2264-2275.

7. Yalti S, Gurbuz B, Ficicioglu C. Serum levels of inhibin B in men and their relationship with gonadal hormones, testicular volume, testicular biopsy results and sperm parameters. J Obstet Gynaecol. 2002;22(6):649-654.

8. Aydinok Y, Bayraktaroglu S, Yildiz D, Alper H. Myocardial iron loading in patients with thalassemia major in Turkey and the potential role of splenectomy in myocardial siderosis. J Pediatr Hematol Oncol. 2011:33(5):374-378.

9. Poggi M, Sorrentino F, Pugliese P, et al. Longitudinal changes of endocrine and bone disease in adults with beta-thalassemia major receiving different iron chelators over 5 years. Ann Hematol. 2016;95(5):757-763.

10. Casale M, Citarella S, Filosa A, et al. Endocrine function and bone disease during long-term chelation therapy with deferasirox in patients with beta-thalassemia major. Am J Hematol. 2014; 89(12):1102-1106. 
11. Singer ST, Vichinsky EP, Gildengorin G, van Disseldorp J, Rosen M, Cedars MI. Reproductive capacity in iron overloaded women with thalassemia major. Blood. 2011;118(10):2878-2881.

12. Chang HH, Chen MJ, Lu MY, et al. Iron overload is associated with low anti-mullerian hormone in women with transfusion-dependent beta-thalassaemia. BJOG. 2011;118(7):825-831.

13. Singer ST, Killilea D, Suh JH, et al. Fertility in transfusion-dependent thalassemia men: effects of iron burden on the reproductive axis. Am J Hematol. 2015;90(9):E190-192.

14. Grunewald S, Glander HJ, Paasch U, Kratzsch J. Age-dependent inhibin B concentration in relation to FSH and semen sample qualities: a study in 2448 men. Reproduction. 2013;145(3):237-244.

15. Oudit GY, Trivieri MG, Khaper N, Liu PP, Backx PH. Role of L-type $\mathrm{Ca} 2+$ channels in iron transport and iron-overload cardiomyopathy. J Mol Med (Berl). 2006;84(5):349-364.

16. Farmaki K, Tzoumari I, Pappa C, Chouliaras G, Berdoukas V. Normalisation of total body iron load with very intensive combined chelation reverses cardiac and endocrine complications of thalassaemia major. Br J Haematol. 2010;148(3):466-475. 